\title{
ROBUST POWER SYSTEM STABILIZER VIA NETWORKED CONTROL SYSTEM
}

\author{
Vojtech VESELÝ — Thuan Nguyen QUANG *
}

\begin{abstract}
The paper presents a novel power system stabilizer (PSS) design for a multivariable power system. The proposed design procedure is based on the linear matrix inequalities and stabilization of controlled system with time-varying time delay.

Keywords: power system stabilizer (PSS), networked control systems (NCSs), time delay, Lyapunov-Krasovskii functional (LKF)
\end{abstract}

\section{INTRODUCTION}

Power system stabilizers are used to enhance damping of power system oscillations mainly through excitation control $[3,5,7-10,13]$. Deregulation of the electricity markets has led to increasing uncertainties concerning the power flow within the network. PSSs are used to enhance damping of power oscillation. In the deregulation electricity market, the phenomenon of poorly damped low frequency inter-area power systems oscillations play an important role and it involves several groups of machines distributed over the different countries. There are several different ways how to improve the oscillation damping in a power system. As shown in references, improvement of inter-area oscillations damping can be advantageously accomplished with PSS. For multivariable power system obviously a typical PSS of the $i$-th synchronous generator (SG) consists of a gain and lead/lag compensation functions with $\pi_{i}$ synchronous generator output feedback. Commonly used PSS, single inputs are shaft speed, terminal frequency, active power (current), terminal voltage and so on. Dual input PSS [9] normally use combinations of power or speed or frequency of the $i$-th SG to derive the stabilizing signal. PSS has decentralized structure.

In this paper, we introduce a novel approach to design of PSS using both classical stabilizing signal with decentralized structure and stabilizing signal which can be obtained from outputs of other SGs that is proposed novel PSS has no decentralized structure. Some idea about such PSS the reader can consults [12]. The data from other units of power system can be obtained using communication data network. Control system over data networks are commonly referred to as Networked Control Systems (NCSs). Integration of communication networks into feedback control loops inevitably leads to some problems. For the NCSs, the sampling data and controller signals are transmitted through a network. As results, it leads to a network-induced delay in a networked control closedloop system. The existence of such a kind of delay in a network-based control loop can induce instability or poor performance of control systems $[2,5,6]$. In this paper, we consider that classical- decentralized stabilizing signal is already defined and problem is to design stabilizing signals obtained from other SGs which guarantee and improve closed-loop system stability, robustness and performances. In this paper, we do not study the problem of which kind of stabilizing signal could be used for complete PSS, but if this stabilizing signal is defined, we study the problem of stability, robustness and performance. The paper is organised as follows. Section 2 gives the preliminaries, definitions and problem formulation. Section 3 explains main results of the paper. And in Section 4, a numerical example is presented to show the design procedure of complete PSS.

Notation: Throughout this paper, for real matrix $M$, the notation $M \geq 0$ (respectively $M>0$ ) means that matrix $M$ is symmetric and positive semi-definite (respectively positive definite); "*" denotes a block that is readily inferred by symmetry; Matrices, if not explicitly stated, are assumed to have compatible dimensions.

\section{PRELIMINARIES AND PROBLEM FORMULATION}

Consider a set of $L$ transfer function matrices identified in several different working points of power system. The working points are determined by steady state of power system with different power, voltages and so on, that are working points cover the whole range of system operation.

$$
G^{k}(s)=\left\{G_{i j}^{k}\right\}_{m \times m}, \quad k=1,2, \ldots, L
$$

where

$$
G_{i j}^{k}(s)=\frac{\Delta \Pi_{i}^{k}}{\Delta U_{j}^{k}}, \quad i, j=1,2, \ldots, m
$$

* Slovak University of Technology, Bratislava, Slovakia vojtech.vesely@stuba.sk, thuan.quang@stuba.sk 


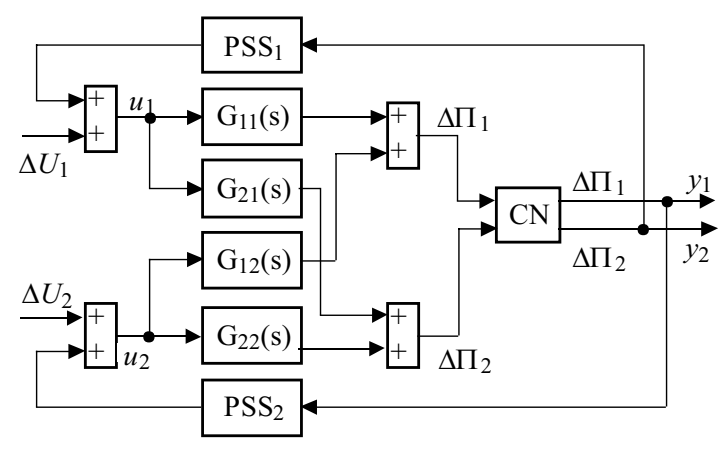

Fig. 1. Structure of complete PSS for the case of $m=2$

$\Delta \Pi_{i}^{k}$ is the change of the $i$-th synchronous generator output for $k$-th experiment applied to the PSS input, $\Delta U_{j}^{k}$ is the change of the demand value of terminal voltage of $j$-th SG. We assume that all synchronous generators operate in the closed-loop with terminal voltage controller and decentralized structure PSS. The structure of SGs and complete PSS for the case of $m=2$ is given in Fig. 1.

For the $P S S(s)$ design procedure, two models are used, [1] Model $M_{1}$ :

$$
P S S(s)=K_{w} \frac{s T_{w}}{1+s T_{w}} \frac{1+s T_{11}}{1+s T_{21}} \frac{1+s T_{12}}{1+s T_{22}} \cdots \frac{1+s T_{1 r}}{1+s T_{2 r}}
$$

where all time constants $T_{j k}$ are known. The problem is to design gain $K_{w}$ and $T_{w}$.

Model $M_{2}$ :

$$
P S S(s)=\frac{s T_{w}}{1+s T_{w}}\left[\begin{array}{lllll}
1 & \frac{1}{1+s T_{2}} & \cdots & \frac{1}{1+s T_{r}}
\end{array}\right]\left[\begin{array}{c}
K_{w 1} \\
K_{w 2} \\
\vdots \\
K_{w r}
\end{array}\right]
$$

Assume that all time constants $T_{j}$ are known. The problem is to design $K_{w 1}, K_{w 2}, \ldots, K_{w r}$ and $T_{w}$.

Combination of the two models different structures of PSS can be obtained. Model of power system (1) should be recalculated to following affine linear time-invariant continuous time uncertain systems.

$$
\begin{aligned}
\dot{x}(t) & =A(\xi) x(t)+B(\xi) u(t), \\
y(t) & =C s(t)
\end{aligned}
$$

where $x(t) \in R^{n}$ is the state vector, $u(t) \in R^{m}$ is the control input of closed-loop system, $y(t) \in R^{l}$ is the output of closed-loop system (Fig. 1). The matrices $A(\xi), B(\xi) \in S$ belong to convex hull, and $S$ is a polytop with $N$ vertices $S_{1}, S_{2}, \ldots, S_{N}$ which can formally defined as

$$
\begin{aligned}
& S:= \\
& \left\{\begin{array}{l}
A(\xi) \in R^{n \times n}, B(\xi) \in R^{n \times m}: A(\xi)=\sum_{i=1}^{N} \xi_{i} A_{i}, \\
B(\xi)=\sum_{i=1}^{N} \xi_{i} B_{i}, \sum_{i=1}^{N} \xi_{i}=1, \xi_{i} \geq 0
\end{array}\right\}
\end{aligned}
$$

where $A_{i}, B_{i}$ are constant matrices with appropriate dimensions and $\xi_{i}$ are time-invariant uncertainties. Note $S$ is a convex and bounded domain.

Consider the single PSS in the following form

$$
P S S(s)=\frac{s T_{w}}{1+s T_{w}}
$$

The problem in this paper is to design two parameters $K$ and $T_{w}$ (structure $\left.M_{1}, M_{2}\right)$ such that for the PSS (7)

$$
u_{i}(s)=\frac{K_{i j} s}{1+s T_{w i j}} e^{-t s} y_{j}(s), i \neq j ; i, j=1,2
$$

where the network induced delay in NCSs is given by $0<\tau \leq \tau_{M}$ and $\dot{\tau} \leq \mu \leq 1$, guarantees the stability, robustness and performance.

In the time domain, the PSS (7) can be reformulated as follow

$$
u(t)=C_{d} W_{f} z(t)+C_{d} K C x(t)-C_{d} K C \int_{t-\tau}^{t} \dot{x}(s) \mathrm{d} s
$$

where

$$
\dot{z}(t)=-W_{f} z(t)+K C x(t)-K C \int_{t-\tau}^{t} \dot{x}(s) \mathrm{d} s
$$

Consider $X(t)=\left[\begin{array}{ll}x^{\top}(t) & z^{\top}(t)\end{array}\right]^{\top}$ we obtain

$u(t)=\left[C_{d} K C-C_{d} W_{f}\right] X(t)+\left[-C_{d} K C \quad 0\right] \int_{t-\tau}^{t} \dot{X}(s) \mathrm{d} s$.

Substituting (10) to (4) will result to the closed-loop system

$$
\dot{X}(t)-A_{c} X(t)+A_{d c} \int_{t-\tau}^{t} \dot{X}(s) \mathrm{d} s=0
$$

where

$A_{c}=\left[\begin{array}{cc}A+B C_{d} K C & -B C_{d} W_{f} \\ K C & -W_{f}\end{array}\right], A_{d c}=\left[\begin{array}{cc}B C_{d} K C & 0 \\ K C & 0\end{array}\right]$.

Note that other part of PSS transfer function can be involved to power system transfer function matrix. The following performance index is associated with closedloop system (4) and (7)

$$
J=\int_{0}^{\infty} J(t) \mathrm{d} t, J(t)=X^{\top}(t) Q X(t)+u^{\top} R u(t)
$$

where $Q=Q^{\top}>0, R=R^{\top}>0$ are matrices of compatible dimensions. Consider

$\eta(t)=\left[\begin{array}{llll}\dot{X}^{\top}(t) & X^{\top}(t) & \int_{t-\tau}^{t} \dot{X}^{\top}(s) \mathrm{d} s & \int_{t-\tau_{M}}^{t-\tau} \dot{X}^{\top}(s) \mathrm{d} s\end{array}\right]^{\top}$ and by substituting $u(t)$ from (10) to $u^{\top}(t) R u(t)$ we obtain

$$
u^{\top} R u(t)=\eta^{\top}(t) K^{\top} R K \eta(t)
$$


where

$$
\begin{gathered}
K=\left[\begin{array}{llll}
0 & K_{1} & K_{2} & 0
\end{array}\right], \\
K_{1}=\left[\begin{array}{lll}
C_{d} K C & -C_{d} W_{f d}
\end{array}\right], \quad K_{2}=\left[\begin{array}{ll}
-C_{d} K C & 0
\end{array}\right]
\end{gathered}
$$

then $J(t)$ can be rewritten as the following form

$$
J(t)=\eta^{\top}(t) M_{Q}(\xi) \eta(t)
$$

where

$$
M_{Q}(\xi)=\left[\begin{array}{cccc}
0 & 0 & 0 & 0 \\
* & K_{1} R K_{1}+Q & K_{1} R K_{2} & 0 \\
* & * & K_{2} R K_{2} & 0 \\
* & * & * & 0
\end{array}\right] .
$$

Associated with the cost of closed-loop system, the guaranteed cost controller is defined as follows:

Definition 1. Consider the uncertain system (4). If there exist a controller of the form (8) and a positive scalar $J_{0}$ such that for all uncertainties (5), the closedloop system (11) is asymptotically stable and closed-loop value of the cost function (12) satisfies $J \leq J_{0}$ then $J_{0}$ is said to be a guaranteed cost and the controller (8) is said to be guaranteed cost controller.

Finally we introduce the well known results from LQ theory.

Lemma 1. Consider the continuous-time delay system (4) with control algorithm (8). The control algorithm (8) is the guaranteed cost control law for the system (4) if and only if there exists $\operatorname{LKF} V(\xi, t)$ such that the following condition holds

$$
\frac{d}{d t} V(\xi, t)+J(t) \leq 0
$$

\section{THE MAIN RESULTS}

The following theorem provides robust stability and robust performance results for the closed-loop system (11).

Theorem 1. Consider the uncertain power system (4) controlled via NCSs with network-induced delay $\tau$ satisfying $0<\tau \leq \tau_{M}, \dot{\tau} \leq \mu \leq 1$ and the cost function (12). If there exist a controller of form (8), scalar $J_{0}$, and matrices $P_{i}>0, G_{i}>0, G_{1 i}>0, G_{2 i}>0(i=1, \ldots, N)$, $N_{1}, N_{2}, N_{3}$ and $N_{4}$ that satisfy the following matrix inequality

$$
W_{i}=\left[\begin{array}{cccc}
w_{i}^{11} & w_{i}^{12} & w_{i}^{13} & w_{i}^{14} \\
* & w_{i}^{22} & w_{i}^{23} & w_{i}^{24} \\
* & * & w_{i}^{33} & w_{i}^{34} \\
* & * & * & w_{i}^{44}
\end{array}\right] \leq 0
$$

where

$$
w_{i}^{11}=N_{1}+N_{1}^{\top}+\tau_{M} G_{1 i}, w_{i}^{12}=-N_{1} A_{c i}+N_{2}^{\top}+P_{i},
$$

$$
\begin{aligned}
w_{i}^{13}= & N_{1} A_{d c i}+N_{3}^{\top}, \quad w_{i}^{14}=N_{4}^{\top}, \\
w_{i}^{22}= & -N_{2} A_{c i}-A_{c i}^{\top} N_{2}^{\top}+\mu G_{i}+K_{1}^{\top} R K_{1}+Q, \\
w_{i}^{23}= & N_{2} A_{d c i}-A_{c i}^{\top} N_{3}^{\top}+(1-\mu) G_{i}+G_{2 i}+K_{1}^{\top} R K_{2}, \\
w_{i}^{24}= & -A_{c i}^{\top}+G_{2 i}, \\
w_{i}^{33}= & N_{3} A_{d c i}-A_{d c i}^{\top} N_{3}^{\top}+(1-\mu) G_{i}-\frac{1}{\tau_{M}} G_{1 i} \\
& -G_{2 i}+K_{2}^{\top} R K_{2}, \\
w_{i}^{34}= & A_{d c i}^{\top} N_{4}^{\top}-G_{2 i}, \\
w_{i}^{44}= & -G_{2 i}-\frac{1}{\tau_{M}} G_{1 i}
\end{aligned}
$$

then the uncertain system (4) with controller (8) is asymptotically stable and the cost function (12) satisfies the following bound

$$
J \leq J_{0}=\sqrt{\lambda_{M P}^{2}+\lambda_{M G}^{2}+\lambda_{M G 1}^{2}+\lambda_{M G 2}^{2}} * J_{M}
$$

where

$$
\begin{aligned}
\lambda_{M P} & =\operatorname{Max}_{i=1 \ldots N}\left(\operatorname{Max}\left(\text { Eigenvalue }\left(P_{i}\right)\right)\right), \\
\lambda_{M G} & =\operatorname{Max}_{i=1 \ldots N}\left(\operatorname{Max}\left(\operatorname{Eigenvalue}\left(G_{i}\right)\right)\right), \\
\lambda_{M G 1} & =\operatorname{Max}_{i=1 \ldots N}\left(\operatorname{Max}\left(\operatorname{Eigenvalue}\left(G_{1 i}\right)\right)\right), \\
\lambda_{M G 2} & =\operatorname{Max}_{i=1 \ldots N}\left(\operatorname{Max}\left(\operatorname{Eigenvalue}\left(G_{2 i}\right)\right)\right), \\
J_{M} & =\left\{\left\|x_{0}\right\|^{4}+\left(\int_{-t}^{0}\|\varphi(s)\|^{2} d s\right)^{2}+\right. \\
& \left.\left(\int_{-\tau}^{0} d \theta \int_{\theta}^{0}\|\dot{\varphi}(s)\|^{2} d s\right)^{2}+\left(\int_{-\tau_{m}}^{-t}\|\varphi(s)\|^{2} d s\right)^{2}\right\}^{1 / 2}
\end{aligned}
$$

where $x(t)=\varphi(t), t \in\left[-\tau_{M}, 0\right]$ is a continuously differentiable initial function.

Pr o of (S k etch). Take the Lyapunov-Krasovskii fucntional as follows

$$
\begin{aligned}
V(\xi, t) & =\sum_{i=1}^{4} V_{i}(\xi, t), \\
V_{1}(\xi, t) & =X^{\top}(t) P(\xi) X(t), \\
V_{2}(\xi, t) & =\int_{t-\tau}^{t} X^{\top}(s) G(\xi) X(s) \mathrm{d} s, \\
V_{3}(\xi, t) & =\int_{-t}^{0} \mathrm{~d} \theta \int_{t+\theta}^{t} \dot{X}^{\top}(s) G_{1}(\xi) \dot{X}(s) \mathrm{d} s . \\
V_{4}(\xi, t) & =\int_{t-\tau_{M}}^{t} X^{\top}(s) G_{2}(\xi) X(s) \mathrm{d} s,
\end{aligned}
$$

Differentiating $V(\xi, t)$ with respect to time and using Newton-Leibnitz formula $x(t-\tau)=x(t)-\int_{t-\tau}^{t} \dot{x}(s) \mathrm{d} s$, we obtain

$$
\dot{V}_{1}(\xi, t)=2 X^{\top}(t) P(\xi) \dot{X}(t),
$$




$$
\begin{aligned}
& \dot{V}_{2}(\xi, t)=\eta_{1}^{\top}(t)\left[\begin{array}{cc}
\mu G(\xi) & (1-\mu) G(\xi) \\
* & -(1-\mu) G(\xi)
\end{array}\right] \eta_{1}(t), \\
& \eta_{2}^{\top}=\left[\begin{array}{ll}
X^{\top} & \int_{t-\tau}^{t} \dot{X}^{\top}(s) \mathrm{d} s
\end{array}\right], \\
& \dot{V}_{3}(\xi, t) \leq \tau_{M} \dot{X}^{\top} G_{1}(\xi) \dot{X}(t)- \\
& \frac{1}{\tau_{M}} \int_{t-\tau}^{t} \dot{X}^{\top}(s) \mathrm{d} s G_{1}(\xi) \int_{t-\tau}^{t} \dot{X}(s) \mathrm{d} s, \\
& \dot{V}_{4}(\xi, t) \leq \eta_{2}^{\top}(t)\left[\begin{array}{ccc}
0 & G_{2}(\xi) & G_{2}(\xi) \\
* & -G_{2}(\xi) & -G_{2}(\xi) \\
* & * & -G_{2}(\xi)
\end{array}\right] \eta_{2}(t) \\
& \eta_{2}^{\top}(t)=\left[\begin{array}{lll}
X^{\top}(t) & \int_{t-\tau}^{t} \dot{X}^{\top}(s) \mathrm{d} s & \int_{t-\tau_{M}}^{t-\tau} \dot{X}^{\top}(s) \mathrm{d} s
\end{array}\right] .
\end{aligned}
$$

Applying the free-weighting matrices technique, equation (8) is represented in the following equivalent form

$$
\begin{aligned}
\alpha(t)=2 \eta^{\top}(t) & {\left[\begin{array}{llll}
N_{1}^{\top} & N_{2}^{\top} & N_{3}^{\top} & N_{4}^{\top}
\end{array}\right]^{\top} } \\
& \times\left[\begin{array}{llll}
M_{d}(\xi) & -A_{c}(\xi) & A_{d c}(\xi) & 0
\end{array}\right] \eta(t)=0 .
\end{aligned}
$$

After manipulation with the above equation, we obtain

$$
\alpha(t)=\eta^{\top}(t) M_{\alpha}(\xi) \eta(t)=0
$$

where

$$
M_{\alpha}(\xi)=\left[\begin{array}{ccc}
N_{1}+N_{1}^{\top} & -N_{1} A_{c}(\xi)+N_{2}^{\top} \\
* & -N_{2} A_{c}(\xi)-A_{c}^{\top}(\xi) N_{2}^{\top} \\
* & * & \\
* & * & \\
& N_{1} A_{d c}(\xi)+N_{3}^{\top} & N_{4}^{\top} \\
& N_{2} A_{d c}(\xi)-A_{c}^{\top}(\xi) N_{3}^{\top} & -A_{c}^{\top}(\xi) N_{4}^{\top} \\
& N_{3} A_{d c}(\xi)+A_{d c}^{\top}(\xi) N_{3}^{\top} & A_{d c}^{\top}(\xi) N_{4}^{\top} \\
& & *
\end{array}\right]
$$

Because of $\alpha(t)=0$, thus

$\dot{V}(\xi, t)=\sum_{i=1}^{5} \dot{V}_{i}(\xi, t)+\alpha(t) \leq \eta^{\top}(t)\left[M_{\alpha}(\xi)+M_{V}(\xi)\right] \eta(t)$

where

$$
M_{V}(\xi)=\left[\begin{array}{ccc}
\tau_{M} G_{1}(\xi) & P(\xi) & 0 \\
* & \mu G(\xi) & (1-\mu) G(\xi)+G_{2}(\xi) \\
* & * & -(1-\mu) G(\xi)-G_{2}(\xi) \\
* & * & * \\
& & 0 \\
& & -G_{2}(\xi) \\
& & -G_{2}(\xi)-\frac{1}{\tau_{M}} G_{1}(\xi)
\end{array}\right] .
$$

Due to Lemma 1, the closed-loop system (6) is robustly asymptotically stable and gives an upper bound (a guaranteed cost) for the cost function (7) if

$$
\dot{V}(\xi, t)+J(t) \leq \eta^{\top}(t) W(\xi) \eta(t) \leq 0 \Longleftrightarrow W(\xi) \leq 0 .
$$

$$
\begin{aligned}
W(\xi)=\sum_{i=1}^{N}=\xi_{i} W_{i}= & M_{\alpha}(\xi)+M_{V}(\xi)+M_{Q}(\xi)= \\
& {\left[\begin{array}{cccc}
w^{11}(\xi) & w^{12}(\xi) & w^{13}(\xi) & w^{14}(\xi) \\
* & w^{22}(\xi) & w^{23}(\xi) & w^{24}(\xi) \\
* & * & w^{33}(\xi) & w^{34}(\xi) \\
* & * & * & w^{44}(\xi)
\end{array}\right], }
\end{aligned}
$$$$
w^{11}(\xi)=\sum_{i=1}^{N} \xi_{i} w_{i} 11=N_{1}+N_{1}^{\top}+\tau_{M} G_{1}(\xi)
$$$$
w^{12}(\xi)=\sum_{i=1}^{N} \xi_{i} w_{i} 12=-N_{1} A_{c}(\xi)+N_{2}^{\top}+P(\xi),
$$$$
w^{13}(\xi)=\sum_{i=1}^{N} \xi_{i} w_{i} 13=N_{1} A_{d c}(\xi)+N_{3}^{\top}
$$$$
w^{14}(\xi)=\sum_{i=1}^{N} \xi_{i} w_{i} 14=N_{4}^{\top}
$$

$$
\begin{aligned}
w^{22}(\xi)= & \sum_{i=1}^{N} \xi_{i} w_{i} 22=-N_{2} A_{c}(\xi)-A_{c}^{\top}(\xi) N_{2}^{\top} \\
& +\mu G(\xi)+K_{1}^{\top} R K_{1}+Q
\end{aligned}
$$

$$
\begin{aligned}
w^{23}(\xi)= & \sum_{i=1}^{N} \xi_{i} w_{i} 23 N_{2} A_{d c}(\xi)-A_{c}^{\top}(\xi) N_{3}^{\top} \\
& +(1-\mu) G(\xi)+G_{2}(\xi)+K_{1}^{\top} R K_{2}, \\
w^{24}(\xi)= & \sum_{i=1}^{N} \xi_{i} w_{i} 24=-A_{c}^{\top}(\xi) N_{4}^{\top}+G_{2}(\xi), \\
w^{33}(\xi)= & \sum_{i=1}^{N} \xi_{i} w_{i} 33=N_{3} A_{d c}(\xi)+A_{d c}^{\top}(\xi) N_{3}^{\top} \\
& -(1-\mu) G(\xi)-\frac{1}{\tau_{M}} G_{1}(\xi)-G_{2}(\xi)+K_{2}^{\top} R K_{2},
\end{aligned}
$$

$w^{34}(\xi)=\sum_{i=1}^{N} \xi_{i} w_{i} 34=A_{d c}^{\top}(\xi) N_{4}^{\top}-G_{2}(\xi)$,

$w^{44}(\xi)=\sum_{i=1}^{N} \xi_{i} w_{i} 44=-G_{2}(\xi)-\frac{1}{\tau_{M}} G_{1}(\xi)$.

For each $W_{i} \leq 0, i=1, \ldots, N$, then $W(\xi)=\sum_{i=1}^{N} \xi_{i} W_{i} \leq 0, \sum_{i=1}^{N} \xi_{i}=1, \xi_{i} \geq 0$. Therefore, $\dot{V}(\xi, t) \leq-J(t) \leq 0 \quad(J(t) \geq 0)$, respectively $J(t) \leq$ $-\dot{V}(\xi, t)$. By integrating $J(t) \leq-\dot{V}(\xi, t)$ we obtain

$$
\begin{gathered}
J \leq-\int_{0}^{\infty} \dot{V}(\xi, t) \mathrm{d} t=V_{0}=X_{0}^{\top} P(\xi) X_{0} \\
\quad+\int_{-\tau}^{0} X^{\top}(s) G(\xi) X(s) \mathrm{d} s \\
+\int_{-\tau}^{0} \mathrm{~d} \theta \int_{\theta}^{0} \dot{X}^{\top}(s) G_{1}(\xi) \dot{C}(s) \mathrm{d} s \\
\quad+\int_{-\tau_{M}}^{0} X^{\top}(s) G_{2}(\xi) X(s) \mathrm{d} s .
\end{gathered}
$$




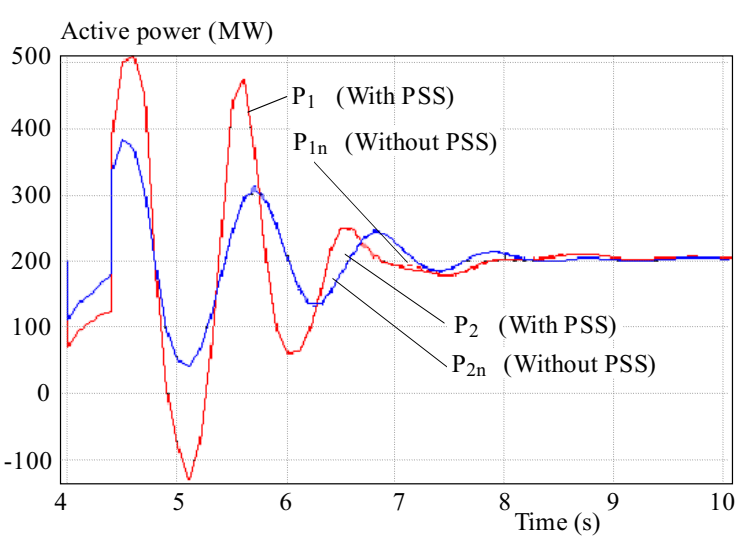

Fig. 2. Regulation of active powers by active power PSS output feedback

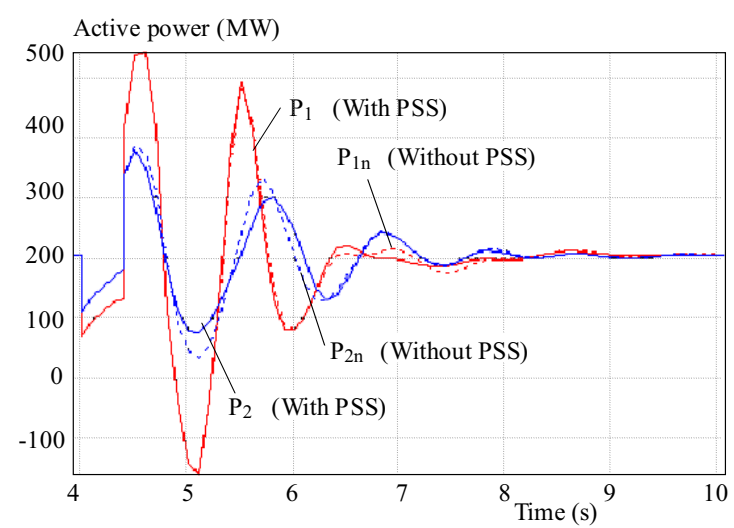

Fig. 3. Regulation of active powers by current PSS output feedback

Because of $X(t)=\left[\varphi^{\top}(t) \quad 0\right], \forall t \in\left[\tau_{M}, 0\right]$ then

$$
\begin{aligned}
& V_{0} \leq \lambda_{M P}\left\|x_{0}\right\|^{2}+\lambda_{M G} \int_{-\tau}^{0}\|\varphi(s)\|^{2} \mathrm{~d} s \\
& \quad+\lambda_{M G 1} \int_{-\tau}^{0} \mathrm{~d} \theta \int_{\theta}^{0}\|\dot{\varphi}(s)\|^{2} \mathrm{~d} s+\lambda_{M G 2} \int_{-\tau_{M}}^{0}\|\varphi(s)\|^{2} \mathrm{~d} s .
\end{aligned}
$$

As we know that, for two arbitrary vectors $\boldsymbol{X}, \boldsymbol{Y}$, the following inequality is always held

$$
\left\|V e c X^{\top} \boldsymbol{Y} \mid \leq\right\| \boldsymbol{X}\|\| \boldsymbol{Y} \|
$$

Consider

$$
\begin{aligned}
\boldsymbol{X} & =\left[\begin{array}{llll}
\lambda_{M P}^{\top} & \lambda_{M G}^{\top} & \lambda_{M G 1}^{\top} & \lambda_{M G 2}^{\top}
\end{array}\right]^{\top} \\
\boldsymbol{Y} & =\left[\begin{array}{lll}
\left\|x_{0}\right\|^{2} & \int_{-\tau}^{0}\|\varphi(s)\|^{2} & \int_{-\tau}^{0} \mathrm{~d} \theta \int_{\theta}^{0}\|\dot{\varphi}(s)\|^{2} \\
& & \int_{-\tau_{M}}^{0}\|\varphi(s)\|^{2} \mathrm{~d} s
\end{array}\right]^{\top} .
\end{aligned}
$$

And applying the inequality (16), the upper bound cost function (7) $J_{0}$ is obtained as (11). Theorem 1. is proved.

\section{EXAMPLE}

Power system stabilizers (PSS) are used to enhance power system damping. The linearized mathematical model of the MIMO power system (1) has been obtained from experiments on the model of the Slovak Power System in the form given by Fig. 1. Experiment has been made for two SG's: EMO11 and EBO31 in one working point, that is $N=1$. For above two SG's obtained transfer function matrix when $\Delta \Pi_{i}=\Delta P_{i}, i=1,2$ - active power of $i$-th SG, has been recalculated to the form of (4), where

$$
\begin{gathered}
A=\left[\begin{array}{cccc}
A_{11} & 0 & 0 & 0 \\
0 & A_{12} & 0 & 0 \\
0 & 0 & A_{21} & 0 \\
0 & 0 & 0 & A_{22}
\end{array}\right], B^{\top}=\left[\begin{array}{cc}
B_{11} & 0 \\
0 & B_{12} \\
B_{21} & 0 \\
0 & B_{22}
\end{array}\right], \\
C^{\top}=\left[\begin{array}{cc}
C_{11} & 0 \\
C_{12} & 0 \\
0 & C_{21} \\
0 & C_{22}
\end{array}\right], D=0,
\end{gathered}
$$

$$
\begin{aligned}
A_{11}= & {\left[\begin{array}{lll}
0 & 0 & -1.2905 \\
1 & 0 & -68.2416 \\
0 & 1 & -1.9819
\end{array}\right], \quad B_{11}=\left[\begin{array}{c}
25.1311 \\
-6.6692 \\
0.8847
\end{array}\right], } \\
A_{12}= & {\left[\begin{array}{lll}
0 & 0 & -106.1244 \\
1 & 0 & -74.3852 \\
0 & 1 & -6.2322
\end{array}\right], \quad B_{12}=\left[\begin{array}{c}
-83.08 \\
100.7547 \\
34.5436
\end{array}\right], } \\
A_{21}= & {\left[\begin{array}{lll}
0 & 0 & -35.2905 \\
1 & 0 & -48.187 \\
0 & 1 & -4.6111
\end{array}\right], \quad B_{21}=\left[\begin{array}{c}
-150.3434 \\
127.6829 \\
4.8710
\end{array}\right], } \\
A_{22}= & {\left[\begin{array}{lll}
0 & 0 & -1.2863 \\
1 & 0 & -68.2668 \\
0 & 1 & -2.4658
\end{array}\right], \quad B_{22}=\left[\begin{array}{c}
10.0213 \\
-3.1781 \\
0.3861
\end{array}\right], } \\
& C_{11}=C_{12}=C_{21}=C_{22}=\left[\begin{array}{ll}
0 & 0 \\
1
\end{array}\right] .
\end{aligned}
$$

Assume that the simple PSS's are used with input active power variable, see Fig. 1. For the following three cases the gains and time constants are calculated: $R=r I, Q=q I, P<p_{\max } I$,

a) $r=1, q=0.01, \tau_{M}=0.001 \mathrm{~s}, \mu=0.1$,

$$
K=\left[\begin{array}{cc}
0.0168 & 0 \\
0 & 0.021
\end{array}\right], T_{w}=\left[\begin{array}{cc}
336.189 & 0 \\
0 & 454.268
\end{array}\right],
$$

b) $r=1, q=0.1, \tau_{M}=0.001 \mathrm{~s}, \mu=0.1$,

$$
K=\left[\begin{array}{cc}
0.0089 & 0 \\
0 & 0.0174
\end{array}\right], T_{w}=\left[\begin{array}{cc}
399.20 & 0 \\
0 & 899.194
\end{array}\right],
$$

c) $r=1, q=0.1, \tau_{M}=0.01 \mathrm{~s}, \mu=0.9$,

$$
K=\left[\begin{array}{cc}
0.0076 & 0 \\
0 & 0.0172
\end{array}\right], T_{w}=\left[\begin{array}{cc}
601.85 & 0 \\
0 & 594.841
\end{array}\right] \text {, }
$$

Dynamic behaviour of the active powers for three phase short circuits and $T=0.4 \mathrm{~s}$, case a) on line V425 are given in Fig. 2. Figure 2 implies that non decentralized structure part of PSS practically does not increase the damper of closed-loop power system dynamic behaviour. 
For the case when $\Lambda \Pi_{i}=I G E N_{i}, i=1,2$ and PSS with transfer function

$$
\begin{aligned}
& P S S_{i}=\frac{k_{i} 0.1 s}{1+0.1 s} \frac{1+0.105 s}{1+1.96 s} \\
& i=1,2, k_{1}=1.76, k_{2}=1.172 .
\end{aligned}
$$

The dynamic behaviours of active powers for three phase short circuits and $T=0.4 \mathrm{~s}$ on line V425 are given in Fig. 3. Figure 3 shows that there exist such SG variables which can be used for complete PSS and such way increases the damper of power system. Conditions of choice such variables are under research.

\section{CONCLUSION}

In this paper, a new approach to the complete robust PSS design has been proposed in time domain. We assume that stabilizing PSS variables are obtained through network communication system. Integration of communication networks into feedback control loops, inevitably leads to some problems.

As results, it leads to a network-induced delay in a networked control closed-loop system. The existence of such a kind of delay in a network-based control loop can induce instability or poor performance of control systems. In this paper sufficient robust stability conditions with guaranteed cost for such system are given. Theoretical results are supported with results obtained by solving robust PSS for two real power units working in Slovak Power System EMO11 and EBO31.

\section{Acknowledgment}

This work has been supported by the Slovak Grant Agency under Grant. No. 1/0544/09.

\section{REFERENCES}

[1] BEFEKADU, G. K.-ERLICH, I. : Robust Decentralized Structure - Constrained Controller Design for Power Systems: An LMI Approach, PSSC, Liege, Aug 2005.

[2] FRIDMAN, E.-NICULESCU, S. J.: On Complete Lyapunov-Krasovskii Functional Techniques for Uncertain Systems with Fast Varying Delays, Int. J. of Robust and nonlinear Control 18 (2008), 364-374.

[3] GRONDIN, R.-KEMWA, I.-SOULIERES, L.-POTRIN, J.-CHAMPEHNE, R. : An Approach to PSS Design for Transient Stability Improvement through Supplementary Damping of the Common Low Frequency, IEEE Trans. Power System 8 No. 3 (1993), 954-963.
[4] GUPTA, R.-BANDYOPADHYAY, B.-KULKARNI, A. M.: Design of Power System Stabilizer for Multimachine Power System using Fast Output Sampling Feedback Technique, ASCC 2002, Proceedings of 4th Asian Control Conference, Singapore, 2002, pp. 1916-1921.

[5] ChARITONOV, V. L.-MELCHELOR-ARQUilAR, D.: On Delay-Dependent Stability Conditions, Systems and Control Letters 40 (2000), 71-76.

[6] CHEN, W. H.-GUAN, Z. H.-LU, X. : Delay-Dependent Output Feedback Guaranteed Cost for Uncertain Time-Delay Systems, Automatica 40 (2004), 1263-1268.

[7] MACHOWSKI, J.-BIALEK, J. W.-ROBAK, S.-BUMBY, J. R.: Excitation Control System for Use with Synchronous Generators, IEE Proc. Gener., Transm. Distrib. 145 No. 5 (1998), 537-546.

[8] MALIK, O. P.-STROYEV, V. A.-STROBEL, V. A.-HANOCK, G. C.-SYROMYATNIKOV, Y. S.: Verification of an Adaptive Excitation Regulator on a Power System Physical Model, Proc. IFAC 13th Triennal World Congress, paper 7d-064, 1996, pp. 187-192.

[9] MURGAŠ, J.-DOBROVIČ, A.-MIKLOVČOVÁ, E.-DÚBRAVSKÝ, J.: Two-Level Turbogenerator Control System, Journal Electrical Eng. 55 No. 3-4 (2004), 83-88.

10] NUMAJIRI, T.-SHIRAI, G.-YOKOYAMA, R.-MACJI, S.-FUJITA, G. : Multimachine Power System Stabilizing Control using Output Feedback Excitation System, Proc. 2003 IFAC Conference Power System Control, 2003, CD-ROM.

[11] PASZEK, S.-PAWLOVSKI, A. : Conference Control of Power System 04, Slovakia, 2004 Dual Input PSS Parameter Optimisation in the Power System with a Nonlinear Mathematical Model, CD-ROM.

12] PASZEK, S.-NOCON, A.: Stabilization of Virtual Power Plant Sources, XXXII IC-SPETO, 2009, CD-ROM.

[13] VESELÝ, V.-KOZÁKOVÁ, A. : Robust PSS Design PSS for a Multivariable Power System, Power Tech IEEE, St.Petersburg, 2005, CD-ROM.

Received 17 September 2010

Vojtech VESELÝ (Prof, Ing, DrSc) was born in 1940. Since 1964 he has worked at the Department of Automatic Control Systems at the Faculty of Electrical Engineering and Information Technology, Slovak University of Technology in Bratislava, where he has supervised $19 \mathrm{PhD}$ students. Since 1986 he has been Full Professor. His research interests include the areas of power system control, decentralized control of large-scale systems, process control and optimization. He is author and coauthor of more than 250 scientific papers.

Thuan Nguyen QUANG born in 1982 in Hanoi, VietNam. Has been studying from 2003 to August 2007 for Bachelor of Science at Faculty of Electrical Engineering and Information Technology, Slovak University of Technology in Bratislava, Slovakia and from 2007 to 2009 for Master of Science at the same place, where he is from 2009 until now doing further studies to get his $\mathrm{PhD}$ title. 